\title{
Instant improvement in monaural spatial hearing abilities through cognitive feedback
}

\author{
Tine Arras ${ }^{1} \cdot$ Hillary Snapp ${ }^{2} \cdot$ Anouk Sangen $^{1} \cdot$ Chantal Snels $^{3,4} \cdot$ Iris Kuntz $^{5} \cdot$ Tinne Theunen $^{6} \cdot$ Kiana Kheirkhah $^{7}$. \\ Andrzej Zarowski $^{6} \cdot$ Thomas Wesarg $^{5} \cdot$ Astrid van Wieringen $^{1} \cdot$ Martijn J. H. Agterberg ${ }^{3,7}$
}

Received: 15 June 2021 / Accepted: 9 February 2022 / Published online: 3 March 2022

(c) The Author(s) 2022

\begin{abstract}
Several studies report that sound localization performance of acute and chronic monauralized normal-hearing listeners can improve through training. Typically, training sessions are administered daily for several days or weeks. While this intensive training is effective, it may also be that monaural localization abilities improve instantly after providing explicit top-down information about the direction dependent change in timbre and level. The aim of the present study was to investigate whether cognitive feedback (i.e., top-down information) could instantly improve sound localization in naive acutely monauralized listeners. Forty-three normal-hearing listeners (experimental group), divided over five different centers, were tested. Two control groups, consisting of, respectively, nine and eleven normal-hearing listeners, were tested in one center. Broadband sounds $(0.5-20 \mathrm{kHz})$ were presented from visible loudspeakers, positioned in azimuth $\left(-90^{\circ}\right.$ to $\left.90^{\circ}\right)$. Participants in the experimental group received explicit information about the noticeable difference in timbre and the poor localization in the monauralized listening condition, resulting in an instant improvement in sound localization abilities. With subsequent roving of stimulus level $(20 \mathrm{~dB})$, sound localization performance deteriorated immediately. The reported improvement is related to the context of the localization test. The results provide important implications for studies investigating sound localization in a clinical setting, especially during closed-set testing, and indicate the importance of top-down information.
\end{abstract}

Keywords Directional hearing $\cdot$ Monaural $\cdot$ Sound level $\cdot$ Timbre $\cdot$ Top-down information

$\begin{array}{ll}\text { Abbreviations } \\ \text { BB } & \text { Broadband } \\ \text { ILDs } & \text { Interaural level differences } \\ \text { ITDs } & \text { Interaural time differences } \\ \text { MAE } & \text { Mean absolute error }\end{array}$

\section{Introduction}

The current study focuses on an underestimated monaural cue (i.e., timbre) arising from the head shadow. When a broadband (BB) sound is presented to the hearing-impaired side of a monauralized listener, the frequency-dependent attenuation provides a physical cue due to the low pass

Communicated by Bill J Yates.

Martijn J. H. Agterberg

m.agterberg@donders.ru.nl; martijnagterberg@gmail.com;

martijn.agterberg@ radboudumc.nl

Extended author information available on the last page of the article filtering by the head, characterized by a change in timbre (Fig. 1). This change in timbre is noticeable when fixed flatspectrum stimuli are presented (Stevens and Newman 1936; Wilska 1938; Wightman and Kistler 1997; Shub et al. 2008).

While many studies address the role of pinna-related spectral cues (e.g., Shub et al. 2008), the noticeable difference in timbre in a monaural listening condition is often overlooked. Timbre can be an important cue and explicit information about the direction dependent change in timbre can have an immediate effect on the monaural listeners' ability to localize sounds. This monaural cue probably plays an important role in clinical studies in which an improvement in sound localization is realized by training (Luntz et al. 2005; Firszt et al. 2015; Bonne et al. 2019).

In the acute monaural hearing condition, binaural processing of interaural time differences (ITDs) and interaural level differences (ILDs) is heavily distorted and listeners perceive the stimuli mainly at the hearing (unplugged) side (Angell and Fite 1901; Musicant and Butler 1984; Oldfield and Parker 1986; Slattery and Middlebrooks 1994; Blauert 1997; Wightman and Kistler 1997; Gordon and Kral 2019). 
Fig. 1 Schematic illustration (adapted from Wilska 1938) indicating the low-pass filtering by the head (subfigure I, red dotted circle). The full spectrum of the signal is perceived at the right ear directed to the sound source (red arrow, subfigure $\mathrm{V})$, while the high frequency components are increasingly attenuated as the positions in azimuth are more distal to the source (subfigures II, III and IV), inducing a change in timbre

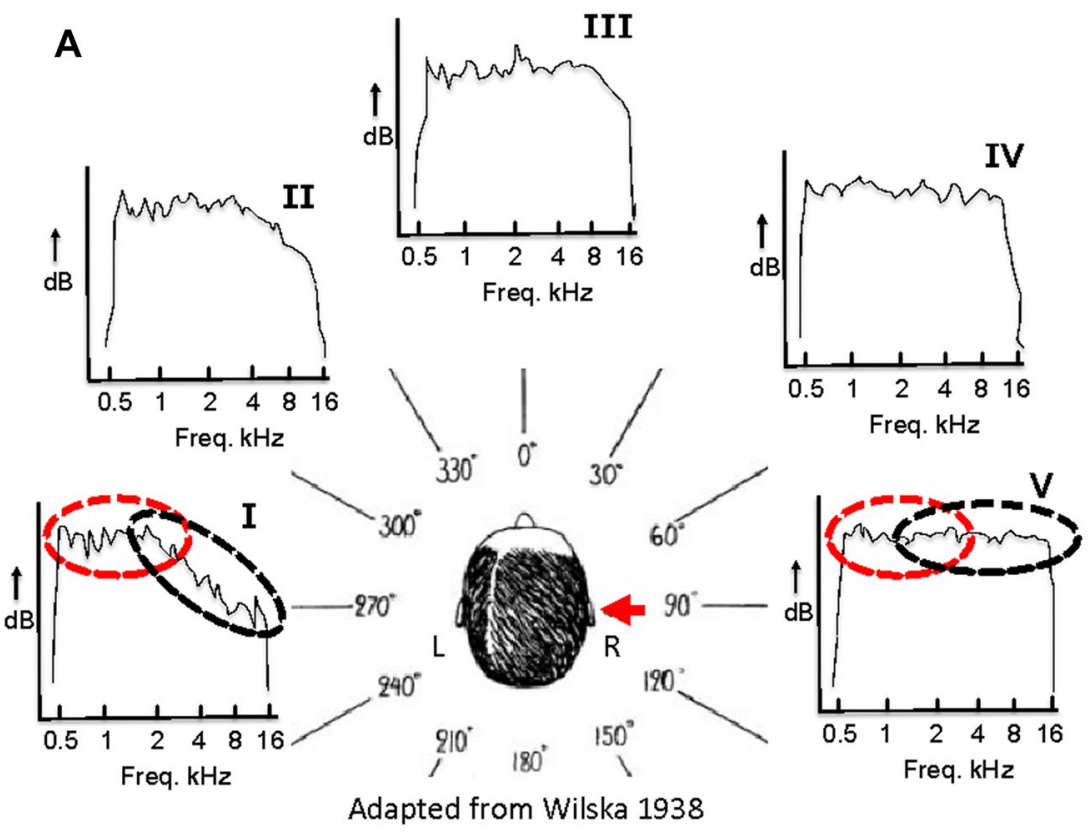

Training can improve sound localization abilities in this acute monaural hearing condition. Typically, several days of training with a few hundred training trials per day were needed to demonstrate an improvement in azimuth localization (Musicant and Butler 1980; Kumpik et al. 2010; Irving and Moore 2011; Strelnikov et al. 2011; Keating et al. 2016; Zonooz and Van Opstal 2019; Rabini et al. 2019; Valzolgher et al. 2020). Notably, during most of these studies participants wore their ear plug only during testing (see Table 1 for study characteristics). Two studies investigated the effect of training in listeners with a chronic unilateral earplug (Kumpik et al. 2010; Irving and Moore 2011).

In general, during training days, feedback from other senses was provided in the form of flashing lights (i.e., visual feedback). These lights indicated the position of the correct loudspeaker or whether a response was correct or incorrect.

Table 1 Characteristics of eight studies investigating the effect of training on free field sound localization of unilaterally plugged normal-hearing listeners

\begin{tabular}{|c|c|c|c|c|c|c|c|c|}
\hline & Earplug & $\begin{array}{l}\text { Number } \\
\text { of sub- } \\
\text { jects }\end{array}$ & Training days & Stimuli & Roving & $\begin{array}{l}\text { Loudspeaker } \\
\text { positions }\end{array}$ & Range & Feedback \\
\hline $\begin{array}{l}\text { Musicant and } \\
\text { Butler (1980) }\end{array}$ & During sessions & 8 & 10 days & Train HP pulses & None & 15 degrees & $90^{\circ}$ & No feedback \\
\hline $\begin{array}{l}\text { Kumpik et al. } \\
\text { (2010) }\end{array}$ & Chronic & 20 & $7-8$ days & $\begin{array}{l}\text { Gaussian noise } \\
(0-20 \mathrm{kHz}), \\
300 \mathrm{~ms}\end{array}$ & $\begin{array}{c}50,56,63,70 \\
77,84 \mathrm{~dB}\end{array}$ & 30 degrees & $360^{\circ}$ & Colored marker \\
\hline $\begin{array}{l}\text { Irving and } \\
\text { Moore (2011) }\end{array}$ & Chronic & 12 & 8 days & $\begin{array}{l}\text { Pink noise, } \\
40,100 \text { and } \\
500 \mathrm{~ms}\end{array}$ & $50-70 \mathrm{~dB}$ & 15 degrees & $360^{\circ}$ & Flashing screen \\
\hline $\begin{array}{l}\text { Strelnikov et al. } \\
\text { (2011) }\end{array}$ & During sessions & 18 & 5 days & $\begin{array}{l}\text { White noise } \\
50 \mathrm{~ms}\end{array}$ & $60 \mathrm{~dB}$ SPL & 10 degrees & $140^{\circ}$ & $\begin{array}{l}\text { Visual "correct" } \\
\text { "incorrect" }\end{array}$ \\
\hline $\begin{array}{l}\text { Keating et al. } \\
(2015)\end{array}$ & During sessions & 11 & $\begin{array}{l}7 \text { sessions } \\
\text { within } \\
3 \text { weeks }\end{array}$ & $\begin{array}{l}\text { Broadband } \\
\text { noise, } 100 \mathrm{~ms}\end{array}$ & 49-77 dB SPL & 30 degrees & $360^{\circ}$ & $\begin{array}{l}\text { Green or Red } \\
\text { flash }\end{array}$ \\
\hline $\begin{array}{l}\text { Rabini et al. } \\
\text { (2019) }\end{array}$ & $\begin{array}{l}\text { During sessions } \\
1,3 \text { and } 5\end{array}$ & 45 & 5 days & $\begin{array}{l}\text { Italian syllable, } \\
500 \mathrm{~ms}\end{array}$ & None & 3 degrees & $43^{\circ}$ & Visual \\
\hline $\begin{array}{l}\text { Zonooz and } \\
\text { Opstal (2019) }\end{array}$ & During sessions & 8 & $>3$ days & High-pass & $50,60,70 \mathrm{~dB}$ & 15 degrees & $120^{\circ}$ & Green LED \\
\hline $\begin{array}{l}\text { Valzolgher et al. } \\
(2020)\end{array}$ & During session & 16 & 3 days & $\begin{array}{l}\text { White-noise, } \\
500 \mathrm{~ms}\end{array}$ & $62 \mathrm{~dB}$ & 7.2 degrees & $120^{\circ}$ & Kinesthetic \\
\hline
\end{tabular}


Several studies demonstrated that feedback from other modalities than the visual system could further improve the monaural localization abilities. For example, kinesthetic cues improve monaural localization abilities when subjects pay attention to the position of sounds (i.e., active training), and these multisensory cues are beneficial for acute monaural listeners (Valzolgher et al. 2020).

There is little agreement on the type of cues, duration of training and method of training needed to improve localization abilities in unilaterally plugged normal-hearing listeners. In clinical setups and in setups in which subjects are trained to improve their localization abilities, loudspeakers are visible and/or the position of loudspeakers is indicated (i.e., closed-set testing). This might result in confounding situations, because participants might perceive sounds from directions that do not correspond with a loudspeaker position. The human neural system is continuously updating available information and unilateral plugged listeners can learn to use monaural cues and pinna-related spectral cues to optimize azimuthal localization in a monaural hearing condition (Wright and Zhang 2006; Kumpik et al. 2010; Keating and King 2015).

In the present study, explicit top-down information, in the form of cognitive feedback, is provided to acutely monauralized listeners to increase the participants' knowledge about the acute monaural listening condition. We hypothesize that this cognitive feedback can instantly improve sound localization abilities in an acute monaural listening condition as this type of feedback assists the learner to reflect on their learning strategies.

Normal-hearing participants $(n=43)$ received a unilateral ear plug and horizontal sound localization was evaluated in four conditions in a within-subject experimental design. Sound localization was tested in the normal-hearing condition (condition 1), in the acute monaural hearing condition before and after receiving explicit information regarding the monaural hearing condition (conditions 2 and 3, respectively), and finally in a condition in which stimuli were presented at three different sound levels (condition 4).

The top-down information consisted of a brief explanation of the monauralized hearing condition and the available monaural cues, combined with a short exposure to the stimuli. Participants were told that all stimuli would be perceived at the side of the open ear, while stimuli were actually presented from all loudspeaker locations. The change in timbre of the stimulus was explained in layman's terms, and participants were exposed to 15 broadband stimuli originating from the five visible loudspeaker positions. Results were compared with two control groups not receiving cognitive feedback.

For clarity, with the terms "cognitive feedback" and "explicit top-down information" we refer to providing contextual information about the acute monaural hearing situation to the participants. The contextual information consisted of two parts. (i) It was explained that they could perceive a change in timbre. (ii) It was told that their performance was poor and that they localized most of the sounds toward the unplugged ear, while stimuli were presented from all loudspeaker locations (i.e., feedback on their poor performance).

\section{Materials and methods}

\section{Participants}

Sixty-two adult participants (about $50 \%$ female, aged $21-57$ years) were included in the study. None of the participants reported a history of inferior hearing or neurological disease, all had normal or corrected to normal vision. Fortythree were tested while providing explicit information (i.e., cognitive feedback), nine were tested in a control condition without theoretical or practical orientation to the task, and eleven were tested without orientation to the task but with exposure to the 15 stimuli presented between condition 2 and 3 . The experiment was conducted at five centers to determine the robustness of the effects, replicability and potential generalization to other sites performing localization assessments. All participants receiving cognitive feedback (Clinic A, $n=10$; Clinic B, $n=10$; Clinic C, $n=10$; Clinic D, $n=6$, Clinic $\mathrm{E}, n=7$ ) and all participants in the control groups (Clinic B, $n=20$ ) were naive to the experimental conditions and had normal-hearing bilaterally as determined by air-conduction hearing thresholds $<20 \mathrm{~dB} H \mathrm{HL}$ across the standard audiometric test frequencies, $250-8000 \mathrm{~Hz}$. All experimental protocols adhered to the guidelines of the universities' local ethics committees.

\section{Control condition}

As a control for the hypothesis two control experiments were performed. Nine normal-hearing participants underwent localization testing under the same experimental conditions as the experimental group, without receiving any top-down information on the monaural hearing condition and without exposure to the $15 \mathrm{BB}$ stimuli between condition 2 and 3 (see Sect. 2.5). In a second control group, eleven normalhearing participants were tested without receiving cognitive feedback but with exposure to the 15 stimuli. These experiments were conducted at the Experimental ORL, under the same test conditions as in ten participants of the experimental group, also assessed at this center. 


\section{Test setups}

The test setups are depicted in Fig. 2. At each site sound localization was tested in a closed-set paradigm in which the loudspeakers were clearly indicated with visual markers. In center $\mathrm{C}$, participants used an indicator box to indicate the loudspeaker number. In centers A, B, D, and E, participants were asked to verbally identify the loudspeaker number. Participants were instructed to maintain their head in a forward position, facing a $0^{\circ}$ azimuth symbol or LED, prior to stimulus presentation, and asked to "head point" to the perceived sound location and indicate the number of the loudspeaker after each stimulus was presented. All sites used a horizontal array with stimuli presented in the frontal hemifield spanning $\pm 90^{\circ}$. A minimum distance of $1 \mathrm{~m}$ between the loudspeakers' front and the center of the participants' head was maintained.

\section{Stimuli and experimental procedure}

All five centers used the same stimulus in all experimental conditions; a $0.5-20 \mathrm{kHz}$ BB Gaussian white noise, custom generated using MATLAB, Version 7.4, The Mathworks, Natick, MA, USA, and saved as WAV file. Stimuli were randomly presented in azimuth. Possible levels were 30, 40 and $50 \mathrm{~dB}$ SL. Prior to testing, SL was determined by plugging and muffing both ears and presenting the $\mathrm{BB}$ stimulus from a location in front of the participant. Stimuli were $150 \mathrm{~ms}$ in duration. Note that because listeners were asked to fix their heads at the center loudspeaker and 150-ms stimuli were presented, it was ensured the participant's head remained stationary during stimulus presentation (Wasmann et al 2020). A total of 94 stimuli were presented during the four conditions. Participants always started with the normalhearing condition, followed by three acute unilateral-plug conditions (see Fig. 3). Half of participants were plugged to the right and the other half to the left. An experimental session lasted approximately $25 \mathrm{~min}$.

\section{Conditions and cognitive feedback}

Localization testing was conducted in a normal-hearing condition (condition 1) and three acute unilateral-plug conditions (conditions 2-4). In the plug conditions one ear was plugged and muffed (simulating acute unilateral hearing loss). Stimuli characteristics, including number and level of stimuli is shown in Fig. 3, along with the source location of the stimuli for each condition.

In condition 1, stimuli were randomly presented at 30 , 40 or $50 \mathrm{~dB}$ SL from each of nine loudspeaker locations, totaling 27 stimuli (Fig. 3).

In condition 2 stimuli were again randomly presented at 30,40 or $50 \mathrm{~dB}$ SL, but from only five loudspeakers, totaling
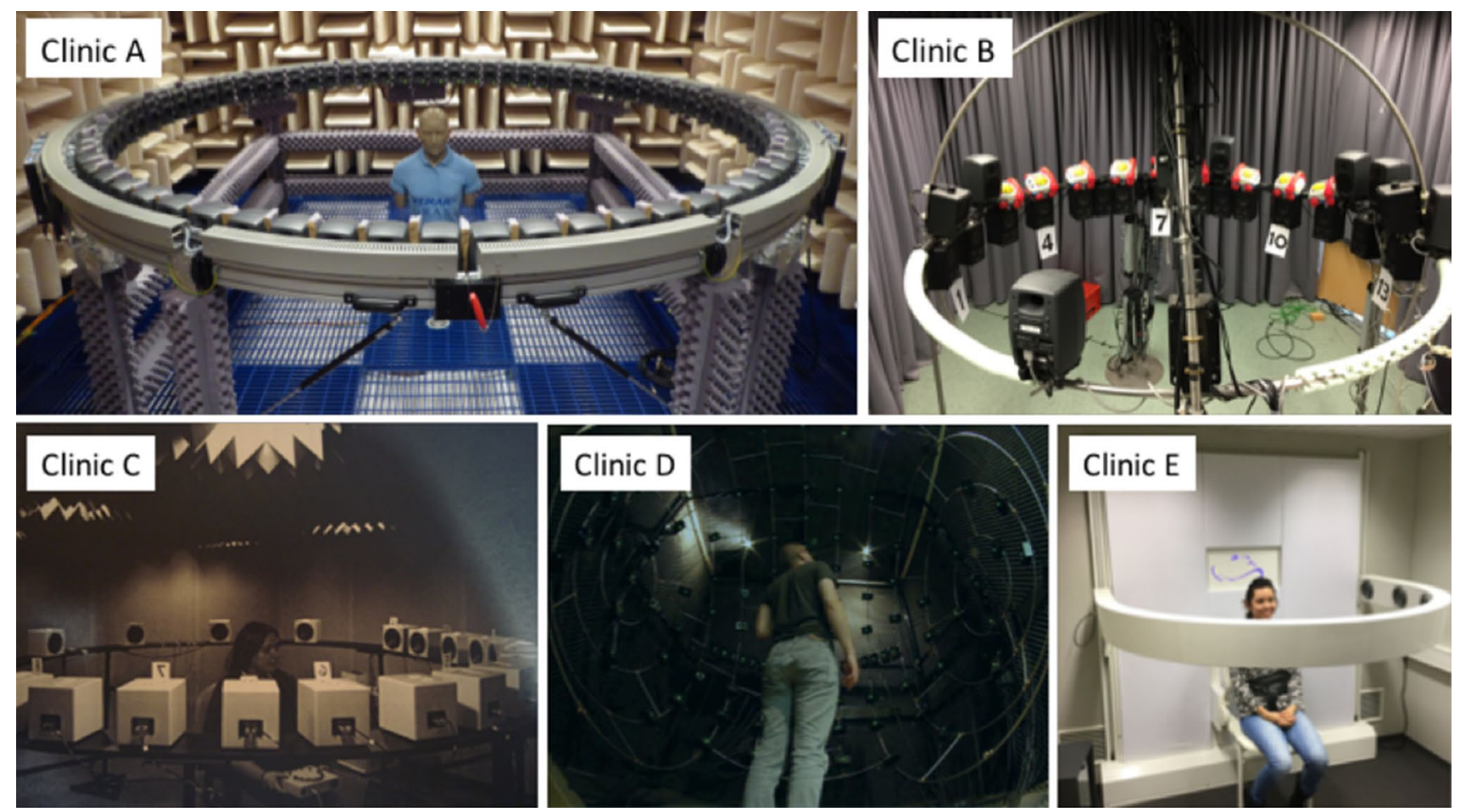

Fig. 2 Illustrations of the different test setups used in the five clinics. All sites used a horizontal array, and at each site the loudspeakers were clearly indicated with a visual marker. At each site, the distance between the loudspeaker and the participant was $1 \mathrm{~m}$ at minimum 


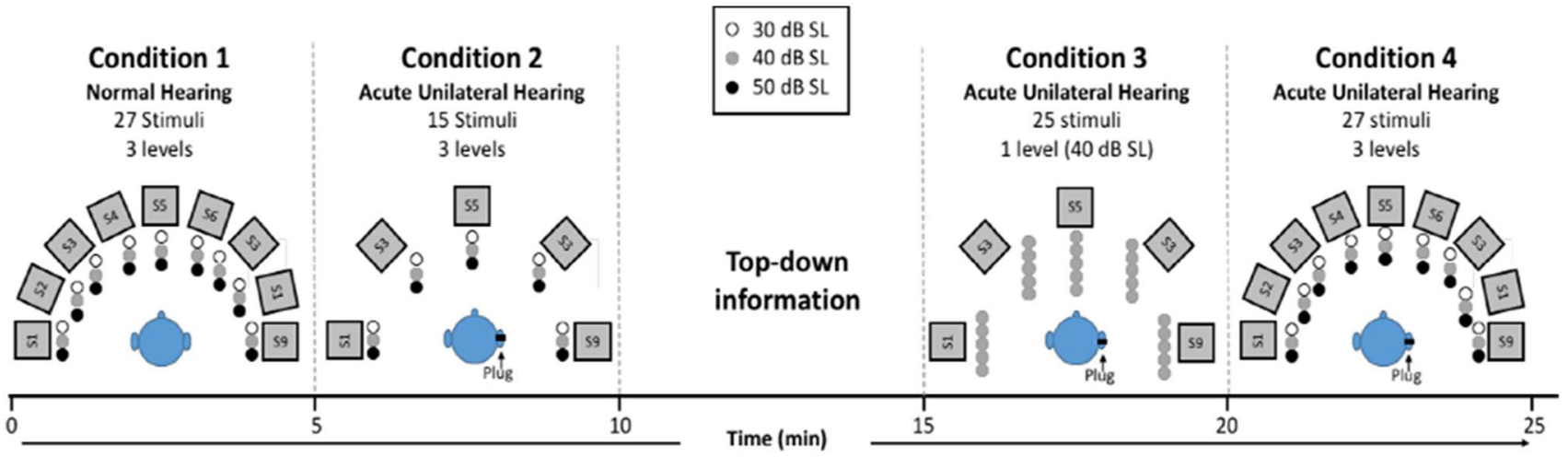

Fig. 3 Schematic representation of the experimental procedure. All stimuli presented in the total experiment are indicated as single dots. Stimulus sound level is indicated with black, gray and white dots. In conditions 2, 3 and 4, participants are unilaterally plugged. The experimental group received cognitive feedback (top-down informa-

15 stimuli (Figs. 4B, 5B and 6B, F). Testing began immediately after plugging one of the ears.

Following condition 2 and immediately prior to condition 3 , the experimental group received top-down information (cognitive feedback) regarding the monaural localization task. Participants were provided with information about their (inaccurate) localization performance demonstrated during the acute unilateral hearing condition (condition 2). Specifically, it was explained that they localized the stimuli, as predicted, mainly towards the side of their normal-hearing (open) ear. Using layman's terms, participants were then informed about the acoustic head-shadow. It was explained that sounds would be perceived different in timbre when originating from different locations. The head acts as a low-pass filter resulting in head-related frequency dependent damping of stimuli originating from the plugged side. tion in about $5 \mathrm{~min}$ ) immediately prior to condition 3 . For the sake of clarity, the small difference in some loudspeaker locations $\left(2.5^{\circ}\right)$ between clinic A and the other clinics, is not indicated. Time scale indicated. $S L$ sensory level

Therefore, broadband noise is more perceived like psss when presented from the hearing side and more like $p s h$ when presented at the side of the plugged ear. Note that this direction-specific subtle change in timbre was already described by Stevens and Newman in 1936. To ensure that the participants understood the provided information, they were exposed to a maximum of 15 stimuli, presented at $40 \mathrm{~dB}$ $\mathrm{SL}$, prior to test condition 3 . These stimuli were presented in sequential order from five clearly indicated loudspeaker positions $\left(90^{\circ}, 45^{\circ}, 0^{\circ},-45^{\circ}\right.$, and $\left.-90^{\circ}\right)$, allowing the subject to perceive the change in loudness and timbre as the signal moved from one side of the head to the other. Altogether, providing participants with information and exposure to the 15 stimuli lasted approximately $5 \mathrm{~min}$.

In condition 3 participants were asked to localize stimuli presented at $40 \mathrm{~dB}$ SL only. Sounds were presented at $40 \mathrm{~dB}$
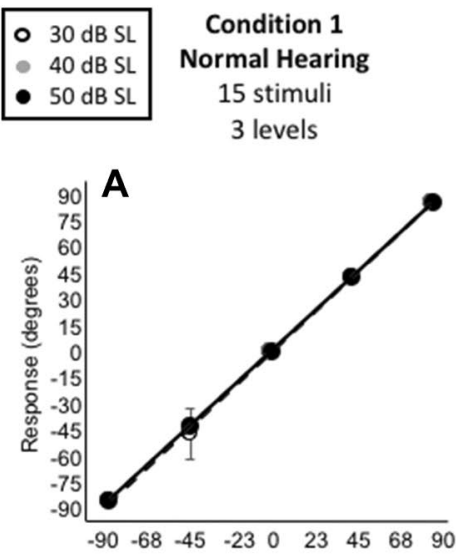

Condition 2

Acute Unilateral Hearing 15 stimuli 3 levels
Condition 3

Acute Unilateral Hearing

25 stimuli

1 level ( $40 \mathrm{~dB} S \mathrm{~L})$

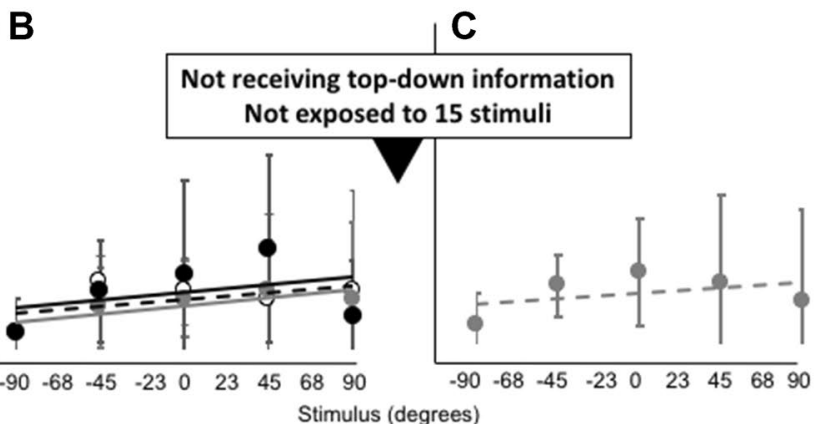

Condition 4

Acute Unilateral Hearing 15 stimuli 3 levels

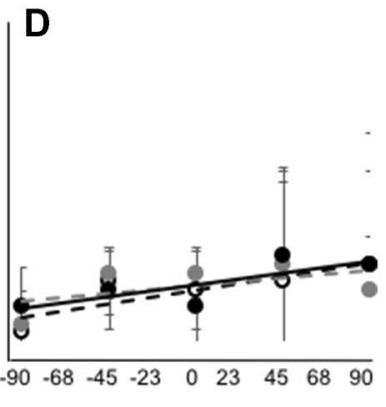

Fig. 4 Average stimulus-response plots ( \pm standard deviation) for nine control listeners who did not receive cognitive feedback (i.e., who did not receive top-down information). Note the remaining clear leftward bias (B-D) 


\begin{tabular}{|c|c|}
\hline - $30 \mathrm{~dB} \mathrm{SL}$ & Condition 1 \\
\hline - $40 \mathrm{~dB}$ SL & Normal Hearing \\
\hline - $50 \mathrm{~dB}$ SL & $\begin{array}{c}15 \text { stimuli } \\
3 \text { levels }\end{array}$ \\
\hline
\end{tabular}

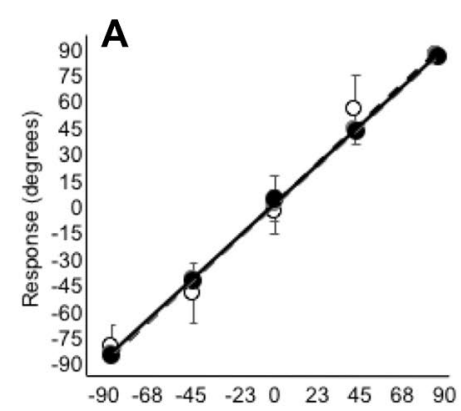

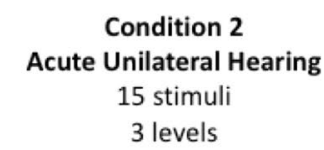

B

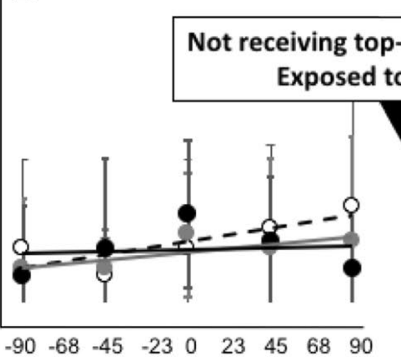

Condition 3

Acute Unilateral Hearing

25 stimuli

1 level (40 dB SL)
Condition 4

Acute Unilateral Hearing

15 stimuli

3 levels

\section{C}

D

Stimulus (degrees)

Fig. 5 Average stimulus-response plots ( \pm standard deviation) for eleven control listeners who were exposed to 15 stimuli between condition 2 and 3 but who did not receive cognitive feedback (i.e., who did not receive top-down information). Note the remaining clear leftward bias (B-D)
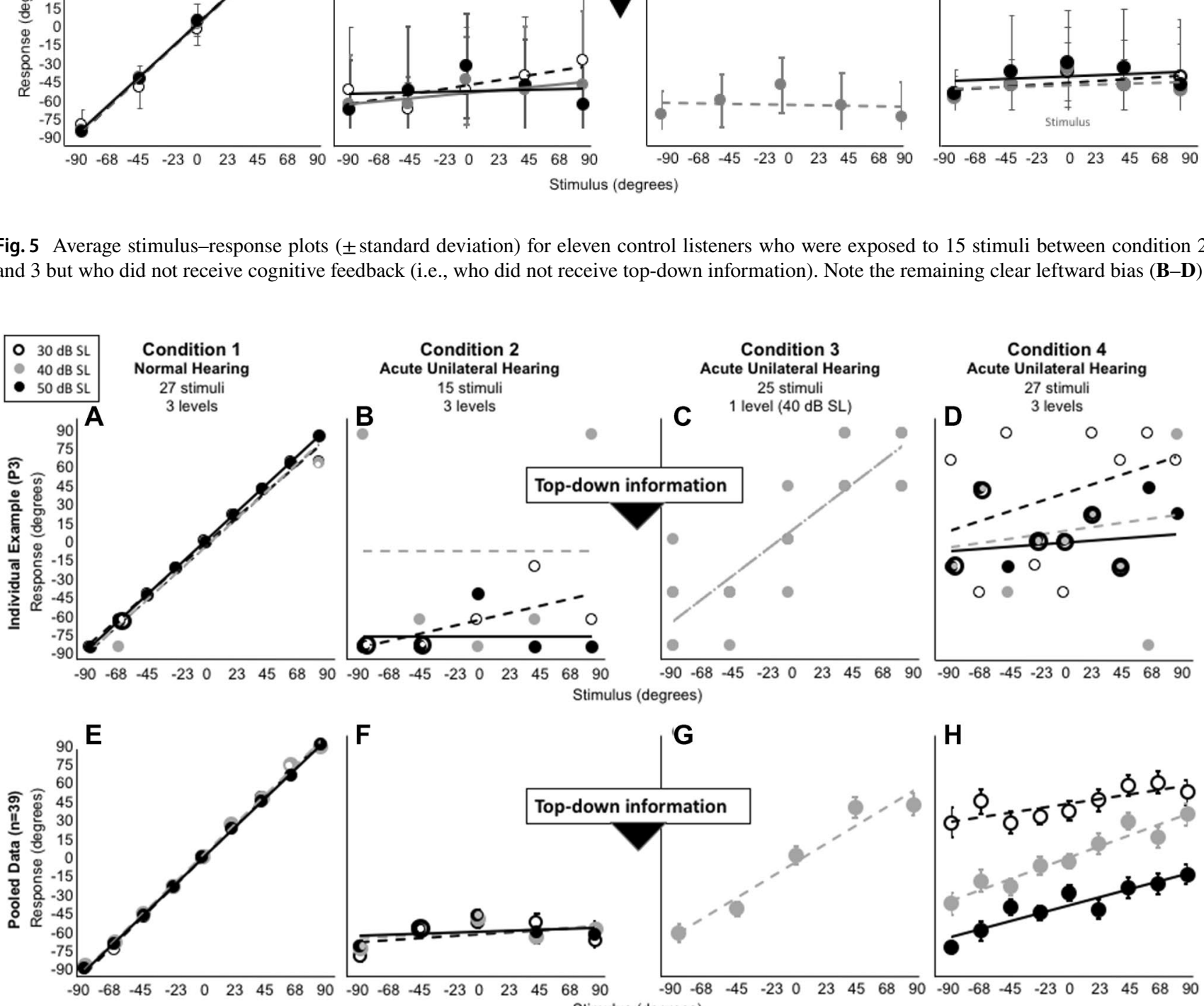

timulus (degrees)

Fig. 6 Individual stimulus-response plots (A-D) and the average stimulus-response plots $( \pm$ standard deviation) in all four conditions for all 39 participants $(\mathbf{E}-\mathbf{H})$. Note the clear leftward bias $(\mathbf{B}, \mathbf{F})$ prior to providing the top-down information, and the improved sound local-

only, because a change in timbre is better detectable when sound level is not roved. Twenty-five stimuli were presented at random from five loudspeaker locations (five stimuli from each loudspeaker).

In condition 4 , the experimental procedure of condition 3 was repeated without informing the participants that ization after providing top-down information $(\mathbf{C}, \mathbf{G})$. When stimuli were presented at three levels $(\mathbf{D}, \mathbf{H})$ sound localization deteriorated immediately. Particularly at group level, a clear level-dependent bias is visible $(\mathbf{H})$

stimuli were randomly presented at 3 levels (30, 40 and $50 \mathrm{~dB}$ SL), and that the number of loudspeaker locations was increased from five back to nine (see Fig. 2). Participants received verbal encouragements without providing any specific information regarding their performance. 


\section{Data analysis}

The individual data $(n=63)$ were checked for consistency and adherence to the protocol. In total, four protocol deviations were identified, resulting in a total of 39 data sets in the experimental group and 20 data sets in the control groups. Individual data were adjusted so that responses corresponded to right-ear plugging and then grouped for analysis. The mean absolute errors of the stimulus-response relations were calculated and analysis of variance (ANOVA) was used to assess the effects.

\section{Results}

Figures 4 and 5 show the pooled stimulus-response relationships for the control participants $(n=9$; Fig. 4 and $n=11$; Fig. 5) for each condition. As expected, target response accuracy was high for condition 1 (Figs. 4A, 5A). Following acute plugging, the control groups demonstrate a strong lateralization towards the open ear for all monauralized listening conditions (Figs. 4B-D, 5B-D). Exposure during condition 3 (Fig. 4C) and 4 (Fig. 4D) did not result in a significant change in localization behavior, demonstrating a continued reliance on highly disturbed binaural cues. Exposure to 15 stimuli between condition 2 and 3 did not affect localization performance (compare Fig. 4C, D with Fig. 5C, D).

Figure 6 shows results of the experimental group $(n=39)$ for each condition. Here, an individual participant example (upper row), and the pooled stimulus-response relationship for all 39 participants (lower row) is presented. The stimulus-response relationship for the normal-hearing condition (Fig. 6A, E) is consistent with that observed in the control groups (Figs. 4A and 5A), and demonstrates the typical diagonal orientation of the regression line indicating accurate localization. ANOVA on mean absolute error (MAE) for the pooled data yielded a significant main effect of condition, $F(3,152)=136.98$, $p<0.0001$. Post hoc comparisons (Table 2) using the Tukey Honest Significant Difference (HSD) test indicated that the MAE under normal-hearing conditions $\left(3.1^{\circ}\right)$ was significantly lower (better) than under any of the simulated hearing loss conditions $\left(64^{\circ}, p<0.0001\right)$. Normal-hearing localization abilities were observed across all levels as demonstrated by a high degree of stimulus-response accuracy.

In the acute unilateral-plug condition (Fig. 6B, F), a strong open-ear bias was observed, reflected by the negative response azimuth values between $-45^{\circ}$ and $-90^{\circ}$ (Fig. 6F, MAE $=64 \cdot 2^{\circ}$ ). Localization in this acute unilateral-plug condition was significantly worse than for normal hearing (Tukey HSD test, $p<0.0001$ ). At the individual level it is visible that stimuli were not always perceived toward the hearing ear (Fig. 6B).

In condition 3 (Fig. $6 \mathrm{C}, \mathrm{G}$ ) the overall MAE $\left(31^{\circ}\right)$ was considerably smaller than for condition $2\left(64^{\circ}\right.$, $p<0.0001)$. Unlike that observed in the controls, a clear improvement and highly accurate localization performance is indicated by the diagonal orientation of the data points (Fig. 6G). The control groups continued to demonstrate a strong response bias toward the open ear (Figs. 4C, 5C).

Figure 6D, $\mathrm{H}$ (condition 4) demonstrates the disruption in localization accuracy that occurred with roving of sound level. Review of grouped data (Fig. $6 \mathrm{H}$ ) shows that stimuli presented at $30 \mathrm{~dB}$ SL were perceived towards the plugged side, and stimuli presented at $50 \mathrm{~dB}$ SL towards the side of the unplugged ear. Interestingly, participants were more accurate in localizing stimuli presented at $40 \mathrm{~dB}$ SL (diagonal orientation of the data), though performance does not reach that which is observed in condition 3 (i.e., comparison grey regression line Fig. $6 \mathrm{G}$ with $6 \mathrm{H}$ ). The data demonstrates that, when the stimuli are roved over a $20-\mathrm{dB}$ range, the monaural cue becomes unreliable.

Figure 7 shows that the experimental group had smaller (better) MAEs in condition 3 than in condition 4 (most points lie above the diagonal), demonstrating that overall sound localization was better in condition 3 compared to condition 4. Furthermore, the figure reveals that the whole data set contains only a few outliers, tested in clinic $\mathrm{C}$, with an MAE $>60^{\circ}$.
Table 2 Multiple comparisons between test conditions

\begin{tabular}{lllllll}
\hline Condition 1 & Condition 2 & Mean Diff & Std Err Diff & Lower CL & Upper CL & $p$ value \\
\hline C1 NH & C2 Acute UH & 61.08 & 3.2 & 52.78 & 69.39 & $<.0001$ \\
& C3 Acute UH & 28.35 & 3.2 & 20.04 & 36.67 & $<.0001$ \\
& C4 Acute UH & 47.55 & 3.2 & 39.24 & 55.86 & $<.0001$ \\
C2 Acute UH & C3 Acute UH & 32.73 & 3.2 & 24.42 & 41.04 & $<.0001$ \\
& C4 Acute UH & 13.53 & 3.2 & 5.22 & 21.85 & $<.0002$ \\
C3 Acute UH & C4 Acute UH & 19.2 & 3.2 & 10.88 & 27.51 & $<.0001$ \\
\hline
\end{tabular}

One-way ANOVA on MAE for the pooled data yielded significant variation among conditions, $F(3$, $152)=136.98, p<0.0001$. Post hoc comparisons using the Tukey HSD test is presented

$U H$ unilateral hearing 


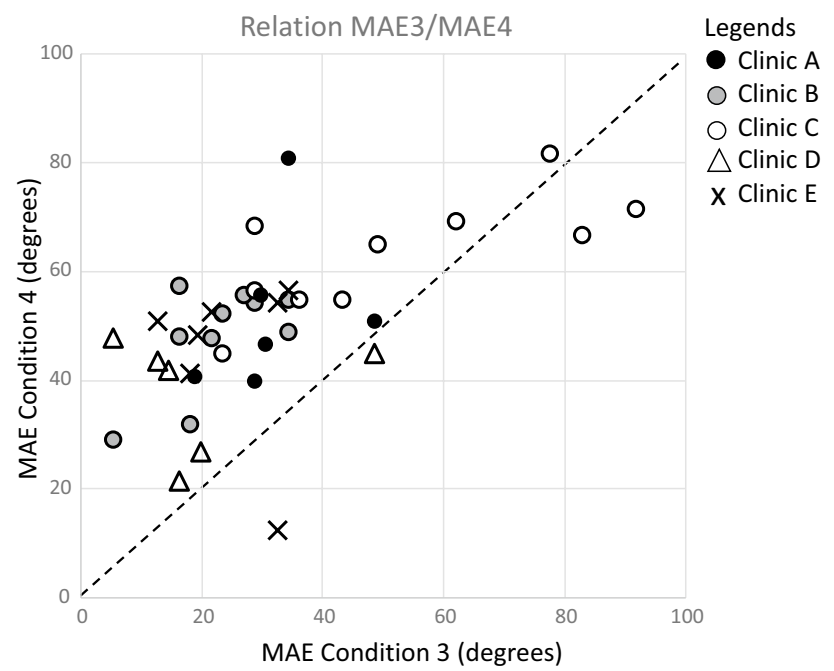

Fig. 7 Relation between MAE obtained in condition 3 and in condition 4 in all 39 participants. Most data points lie above the diagonal, indicating better localization abilities in condition 3 compared to condition 4

\section{Discussion}

\section{Instant improvement in sound localization}

The present study shows that providing participants $(n=39)$ with explicit top-down information about the acute monaural hearing situation, in combination with minimal exposure to 15 BB noise bursts, instantly improved monaural localization abilities. Introducing roving of sound level re-disrupted monaural localization (Fig. 6H). Nine and eleven participants, tested under the same experimental conditions without receiving cognitive feedback (Fig. 4), and with exposure to 15 stimuli (Fig. 5), did not demonstrate any improvement in localization.

\section{Training}

The eight studies listed in Table 1 demonstrated improved monaural localization abilities after training (Musicant and Butler 1980; Kumpik et al. 2010; Irving and Moore 2011; Strelnikov et al. 2011; Keating et al. 2016; Zonooz and Van Opstal 2019; Rabini et al. 2019; Valzolgher et al. 2020). These studies showed a practice effect and the reweighting of monaural spectral information. Kumpik et al. (2010) demonstrated that, after daily training sessions with visual feedback for at least 1 week, monauralized normal-hearing listeners acquire the ability to use monaural spectral cues for the localization of sounds in azimuth. Furthermore, they demonstrated that there was no clear evidence for adaptation to altered ITDs and ILDs. Interestingly, recent studies demonstrated that simple test-retesting, without providing the visual feedback, resulted in an improvement in monaural localization abilities (Rabini et al. 2019; Zonooz and Van Opstal 2019).

Typically, in all the studies mentioned above, participants were exposed to a large number of stimuli during the training and testing sessions (>1300 stimuli). Only Musicant and Butler (1980) adopted a procedure in which participants received as limited as possible additional auditory experience in their setup. Still, participants were exposed to 660 stimuli. Note that in the present study participants were exposed to only 109 stimuli (duration experiment about $25 \mathrm{~min}$ ).

Recently, several studies showed that sensory feedback from other modalities than the visual system could further improve sound localization (Fletcher et al. 2020; Valzolgher et al. 2020). For example, a striking improvement in monaural localization is demonstrated in participants who moved an audio-bracelet, attached to their wrist, while paying attention to the direction-dependent sounds emitted by this audio bracelet (Valzolgher et al. 2020).

These above-mentioned studies differ in many aspects from each other (see Table 1), and it remains unclear which (monaural) cues are dominant. A general model, adapted from the conceptual model (Blauert 1997; Hofman and Van Opstal 1998; Zonooz and Van Opstal 2019), indicates how top-down information and information from other senses can heavily affect the processing of altered binaural cues (Fig. 8). The model extends previous models describing factors within the auditory system (Hartmann et al. 1998a; Braasch 2016) by adding the contribution of timbre. In the normal-hearing condition (Fig. 8A) sound localization is optimal because of the accurate processing of binaural cues (i.e., ITDs and ILDs). This is indicated by the bold solid lines (I) and is reflected by the accurate localization in condition 1 (Figs. 4A, 5A and 6E). In the acute monaural hearing condition (Fig. 8B), the ITDs and ILDs are highly disturbed, indicated by the 'bold dashed' and 'bold solid' line (I), resulting in a strong bias towards the hearing ear because of an extreme ILD (see condition 2, Figs. 4B, 5B and 6F). The present study demonstrates the instant improvement in condition 3 (Fig. 6G) based on the use of timbre and level after providing cognitive feedback (bold solid lines, Fig. 8C). Training with visual and/or haptic feedback (Irving and Moore 2011; Strelnikov et al. 2011; Oldegaard et al. 2015; Fletcher et al. 2020; Valzolgher et al. 2020) can change the behavior resulting in reasonable localization abilities when stimuli are presented at one single level, and several studies indicated that monauralized listeners can learn to use spectral pinna cues (i.e., HRTFs) for localization of sounds in azimuth (Kumpik et al. 2010; Keating et al. 2016) indicated by the 'bold solid' line in Fig. 8D. Note that this post-training effect is not part of the current study. 
Fig. 8 Schematic drawing of a general model indicating the dominant mechanisms underlying the performance in the normal-hearing condition (A), acute monaural hearing condition $(\mathbf{B})$, acute monaural hearing condition after cognitive feedback $(\mathbf{C})$ and the post-training monaural hearing condition (D). Potentially, four acoustic cues (I, II, III and IV) can contribute to localization in azimuth. In the monauralized conditions several cues and or factors can become dominant. ITD Interaural time differences, $I L D$ Interaural level differences, $H R T F$ head-related transfer functions
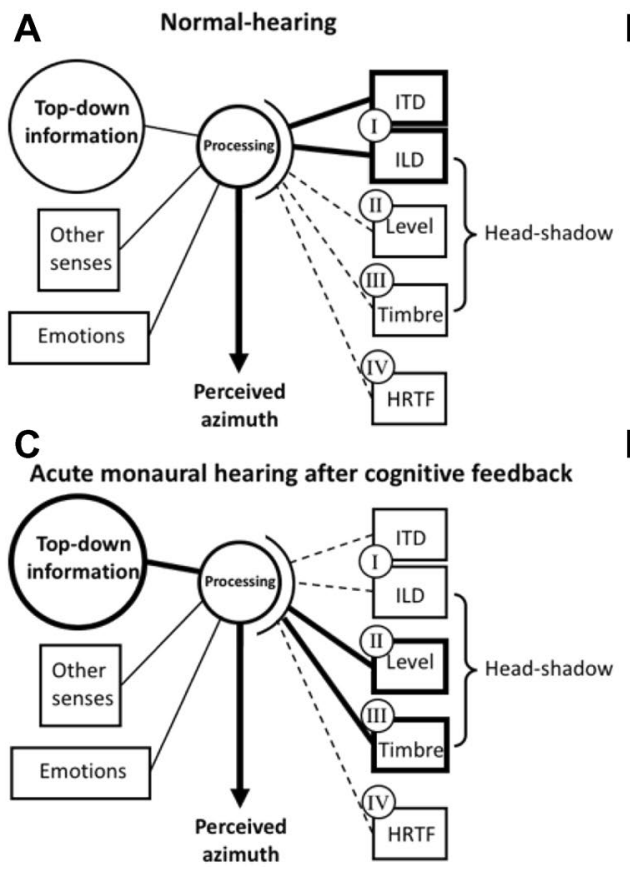

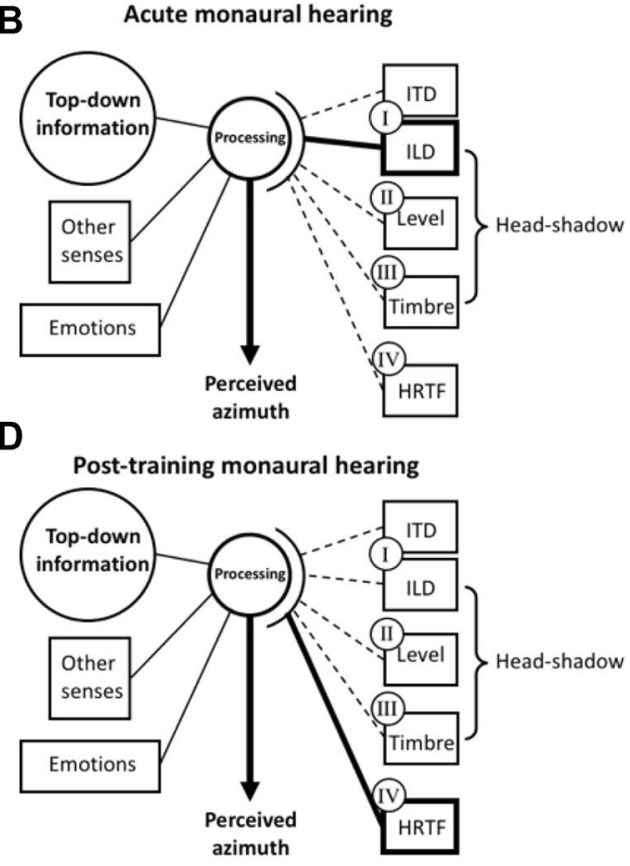

The presented model displays the multidisciplinary aspects of the presented work. The contribution of top-down processing in spatial hearing is important, although not yet fully understood (Souffi et al. 2021). Studying "cognitive feedback" in this field of research is complex and there is still a lot to gain, since deep knowledge in psychology, metacognition (Flavell 1979; Dunlosky and Hertzog 2000), neuroscience and audiology is needed to really understand which factors are crucial in training sound localization abilities. For example, when participants are unaware that the conditions are changed while being tested, knowledge updating cannot occur. Monitoring their accuracy during the test, metacognitive experience and the ability to test assumptions can all have significant effects on their localization performance.

\section{Possible mechanisms underlying monaural localization abilities}

In many clinical studies, and in studies with unilaterally plugged normal-hearing listeners, it remains uncertain which of the possible (auditory) cues are key in the reported improvements. Especially in plugged normalhearing listeners (Wightman and Kistler 1997) but also in patients with unilateral aural atresia (Agterberg et al. 2012; Kumpik and King 2019; Thompson et al. 2020; Canfarotta et al. 2021) remnant binaural cues can explain accurate localization of high-level sounds. Apparently, remnant binaural cues are not important in the presented acute monaural condition, since all stimuli are perceived extremely towards the open ear (Figs. 4B, 5B, F). In the present study, unbeknownst to the listener, during the last test condition the signal was roved over three levels spanning a 20-dB range (Fig. 3). Compared to the test condition in which the stimuli were not roved, overall localization deteriorated, with the overall MAE increasing from $31^{\circ}$ in condition 3 to $51^{\circ}$ in condition $4(p<0.0001)$. However, localization for the $40 \mathrm{~dB}$ stimuli rarely differed between both conditions. The results demonstrate that when the level is roved, using sound level becomes an inaccurate strategy, resulting in a bias towards the open normal-hearing ear for high-level stimuli and a bias towards the plugged ear for low-level stimuli (Fig. 6H). In contrast, this effect of sound level was not present for the control groups who did not receive any top-down information regarding the monauralized listening condition (Figs. 4D, 5D).

The demonstrated instant improvement is related to the context of the localization test. Top-down information regarding the sound locations and knowing that sounds are presented at one sound level provided useful information that can be used instantly. When the levels are roved sound localization deteriorates. In this experiment this deterioration is related to the fact that the participants were not aware that we changed the experimental condition. In other words, the reported improvement is related to the context of the experiment and does not necessarily reflect an improvement in localization skills, and more research is needed to investigate generalization of results obtained in laboratory settings to real-world outcomes (Risoud et al. 2019). 


\section{Factors affecting sound localization}

When investigating sound localization abilities, the sound level is often not roved (Bosman et al. 2001; Luntz et al. 2005; Kitterick et al. 2011; Hansen et al. 2013; Kuhnle et al. 2013; Litovsky et al. 2013; Monini et al. 2015; Parisa et al. 2017; Asp et al. 2018; Eklof et al. 2018; Yang et al. 2018; Bonne et al. 2019; Zirn et al. 2019; Valzolgher et al. 2020), or only roved over a small $(<10 \mathrm{~dB})$ range (Van Deun et al. 2010; Murphy et al. 2011; Nawaz et al. 2014; Firszt et al. 2015; Snapp et al. 2017; Gawliczek et al. 2018; Fletcher et al. 2020). In the absence of sufficient roving, listeners can learn to use the overall level as a cue (Middlebrooks and Green 1991). Moreover, with insufficient roving (i.e., roving $<20 \mathrm{~dB}$ ) of broadband stimuli a salient change in timbre can be used as cue (Wightman and Kistler 1997; Shub et al. 2008). It would be of interest to test the localization ability of acute monauralized normal-hearing listeners with an additional mold in the pinna of the hearing ear. It is expected that in this condition the participants would still demonstrate the instant improvement in localization abilities. In contrast, when participants would suffer a high frequency hearing loss an improvement is not expected, because listeners with presbycusis do not perceive the change in timbre.

To what extent factors such as signal bandwidth (Butler 1986), stimulus level (Macpherson and Middlebrooks 2000; Sabin et al. 2005), visibility of loudspeakers, response method (Populin et al. 2008; Bahu et al. 2016), subjective certainty (Rabini et al. 2020), head movements (Pastore et al. 2020), age (Freigang et al. 2014), sensory (Oldegaard et al. 2015) and motor related input (Valzolgher et al. 2020), pinna cues (Batteau 1967; Shub et al. 2009), reflecting surfaces (Hartmann et al. 1998), experimental design, and top-down information contribute to acute and chronic monaural sound localization abilities requires further study. Furthermore, it would be of interest to study the different aspects of the topdown and bottom-up information in more detail.

Regarding the influence of vision and visibility of the loudspeakers: when (loud)speakers are visible, visual cues might dominate usage of ILDs and ITDs. An example is the strong ventriloquism effect (Hendrickx et al. 2015). Because of the dominance of visual cues in some listening situations, processing of ITDs and ILDs is typically investigated in complete darkness. However, assessment of sound localization abilities in a clinical setting is often performed using setups with visible loudspeakers.

Regarding the response method: it is well known that methodological differences in target pointing can affect the accuracy of a subject's response (Bahu et al. 2016). The present data (Fig. 7) suggest that an indirect pointing method with an indicator box (clinic $\mathrm{C}$ ) results in less accurate responses compared to simply head-oriented responses (all other clinics).
Regarding the cognitive feedback: participants were told that they performed poorly in condition 2 and that they localized most of the sounds toward the unplugged ear, while stimuli were presented from all speaker locations. This information by itself, without any additional information regarding the change in timbre, could have affected performance. Therefore, the nature of the manipulation requires further research.

\section{Conclusion}

We demonstrate that localization abilities of monauralized normal-hearing listeners improved immediately after providing explicit information about the acute monaural hearing condition, when BB sounds were presented at a single stimulus level. The ability to improve monaural localization was not affected by small differences between the different test sites with regard to sound-localization setups, demonstrating how robust and generalizable the observed effect is.

We conclude that providing top-down information regarding the acute monaural listening situation in combination with information regarding the change in timbre that can be perceived in the monaural condition, instantly improves the localization abilities when loudspeakers are visible during closed-set testing. The results have important clinical implications and should be considered when investigating sound localization abilities, and when providing training, after treatment of (unilaterally) hearing-impaired patients.

Acknowledgements This research was funded by the William Demants og Hustru Ida Emilie's Fond (MA, 16-0042).

Author contribution All authors contributed to the study, with: TA, AvW, HS and MA writing the initial draft of the manuscript, AS and MA designing the experiment and conceptualization; AS, AZ, CS, HS, TA, IK and TT mainly collecting the data; AS, CS and HS analyzing the data; TA, AvW, HS, TW, KK and MA writing, reviewing and editing the manuscript. No conflict of interest is reported and the authors do not have financial interest in the subject matter.

Open Access This article is licensed under a Creative Commons Attribution 4.0 International License, which permits use, sharing, adaptation, distribution and reproduction in any medium or format, as long as you give appropriate credit to the original author(s) and the source, provide a link to the Creative Commons licence, and indicate if changes were made. The images or other third party material in this article are included in the article's Creative Commons licence, unless indicated otherwise in a credit line to the material. If material is not included in the article's Creative Commons licence and your intended use is not permitted by statutory regulation or exceeds the permitted use, you will need to obtain permission directly from the copyright holder. To view a copy of this licence, visit http://creativecommons.org/licenses/by/4.0/. 


\section{References}

Agterberg MJ, Snik AF, Hol MK et al (2012) Contribution of monaural and binaural cues to sound localization in listeners with acquired unilateral conductive hearing loss: improved directional hearing with a bone-conduction device. Hear Res 286:9-18

Angell JR, Fite W (1901) Monaural localization of sound. Science 13:775-777

Asp F, Jakobsson AM, Berninger E (2018) The effect of simulated unilateral hearing loss on horizontal sound localization accuracy and recognition of speech in spatially separate competing speech. Hear Res 357:54-63

Bahu H, Carpentier T, Noisternig M et al (2016) Different egocentric pointing methods for 3D sound localization experiments. Acta Acust Acust 102:107-118

Batteau DW (1967) The role of the pinna in human localization. Proc R Soc Lond B Biol Sci 168:158-180

Blauert J (1997) Spatial hearing. The psychophysics of human sound localization. MIT, Cambridge

Bonne NX, Hanson JN, Gauvrit F et al (2019) Long term evaluation of sound localization in single sided deaf adults fitted with a BAHA device. Clin Otolaryngol 2:2

Bosman AJ, Snik AF, van der Pouw CT et al (2001) Audiometric evaluation of bilaterally fitted bone-anchored hearing aids. Audiology 40:158-167

Braasch J (2016) Sound localization in the presence of multiple reflections using a binaurally integrated cross-correlation/auto-correlation mechanism. J Acoust Soc Am. 140:143

Butler RA (1986) The bandwidth effect on monaural and binaural localization. Hear Res 21:67-73

Canfarotta MW, Kane SLG, Buss E (2021) Effects of presentation level on spatial hearing with and without bone-conduction amplification in congenital unilateral aural atresia. Otol Neurotol 42:e388-e392

Dunlosky J, Hertzog C (2000) Updating knowledge about encoding strategies: a componential analysis of learning about strategy effectiveness from task experience. Psychol Aging 15:462-474

Eklof M, Tideholm B (2018) The choice of stimulation strategy affects the ability to detect pure tone inter-aural time differences in children with early bilateral cochlear implantation. Acta Otolaryngol 138:554-561

Firszt JB, Reeder RM, Dwyer NY et al (2015) Localization training results in individuals with unilateral severe to profound hearing loss. Hear Res 319:48-55

Flavell JH (1979) Metacognition and cognitive monitoring: a new area of cognitive-developmental inquiry. Am Psychol 34:906-911

Fletcher MD, Cunningham RO, Mills SR (2020) Electro-haptic enhancement of spatial hearing in cochlear implant users. Sci Rep 10:1621

Freigang C, Schmiedchen K, Nitsche I et al (2014) Free-field study on auditory localization and discrimination performance in older adults. Exp Brain Res 232:1157-1172

Gawliczek T, Munzinger F, Anschuetz L et al (2018) Unilateral and bilateral audiological benefit with an adhesively attached, noninvasive bone conduction hearing system. Otol Neurotol 39:1025-1030

Gordon K, Kral A (2019) Animal and human studies on developmental monaural hearing loss. Hear Res 380:60-74

Hansen MR, Gantz BJ, Dunn C (2013) Outcomes after cochlear implantation for patients with single-sided deafness, including those with recalcitrant Meniere's disease. Otol Neurotol 34:1681-1687

Hartmann WM, Rakerd B, Gaalaas JB (1998) On the source-identification method. J Acoust Soc Am 104:3546-3557
Hendrickx E, Paquier M, Koehl V, Palacino J (2015) Ventriloquism effect with sound stimuli varying in both azimuth and elevation. J Acoust Soc Am 138:3686-3697

Hofman PM, Van Opstal AJ (1998) Spectro-temporal factors in two-dimensional human sound localization. J Acoust Soc Am 103:2634-2648

Irving S, Moore DR (2011) Training sound localization in normal hearing listeners with and without a unilateral ear plug. Hear Res 280:100-108

Keating P, King AJ (2015) Sound localization in a changing world. Curr Opin Neurobiol 35:35-43

Keating P, Rosenior-Patten O, Dahmen JC et al (2016) Behavioral training promotes multiple adaptive processes following acute hearing loss. Elife 5:e12264

Kitterick PT, Lovett RE, Goman AM et al (2011) The AB-York crescent of sound: an apparatus for assessing spatial-listening skills in children and adults. Cochlear Implants Int 12:164-169

Kuhnle S, Ludwig AA, Meuret S et al (2013) Development of auditory localization accuracy and auditory spatial discrimination in children and adolescents. Audiol Neurootol 18:48-62

Kumpik DP, King AJ (2019) A review of the effects of unilateral hearing loss on spatial hearing. Hear Res 372:17-28

Kumpik DP, Kacelnik O, King AJ (2010) Adaptive reweighting of auditory localization cues in response to chronic unilateral earplugging in humans. J Neurosci 30:4883-4894

Litovsky RY, Ehlers E, Hess C, Harris S (2013) Reaching for sound measures: an ecologically valid estimate of spatial hearing in 2- to 3 -year-old children with bilateral cochlear implants. Otol Neurotol 34:429-435

Luntz M, Brodsky A, Watad W et al (2005) Sound localization in patients with unilateral cochlear implants. Cochlear Implants Int 6:1-9

Macpherson EA, Middlebrooks JC (2000) Localization of brief sounds: effects of level and background noise. J Acoust Soc Am 108:1834-1849

Middlebrooks JC, Green DM (1991) Sound localization by human listeners. Annu Rev Psychol 42:135-159

Monini S, Musy I, Filippi C et al (2015) Bone conductive implants in single-sided deafness. Acta Otolaryngol 135:381-388

Murphy J, Summerfield AQ, O’Donoghue GM et al (2011) Spatial hearing of normally hearing and cochlear implanted children. Int J Pediatr Otorhinolaryngol 75:489-494

Musicant AD, Butler RA (1980) Monaural localization: an analysis of practice effects. Percept Psychophys 28:236-240

Musicant AD, Butler RA (1984) The psychophysical basis of monaural localization. Hear Res 14:185-190

Nawaz S, McNeill C, Greenberg SL (2014) Improving sound localization after cochlear implantation and auditory training for the management of single-sided deafness. Otol Neurotol 35:271-276

Odegaard B, Wozny DR, Shams L (2015) Biases in visual, auditory, and audiovisual perception of space. PLoS Comput Biol. 11:e1004649

Oldfield SR, Parker SP (1986) Acuity of sound localisation: a topography of auditory space. III Monaural Hearing Conditions. Perception 15:67-81

Parisa A, Reza NA, Jalal SS et al (2017) Horizontal localization in simulated unilateral hearing loss. J Audiol Otol 22:39-44

Pastore MT, Natale SJ, Clayton C et al (2020) Effects of head movements on sound-source localization in single-sided deaf patients with their cochlear implant vn versus off. Ear Hear 41:1660-1674

Populin LC (2008) Human sound localization: measurements in untrained, head-unrestrained subjects using gaze as a pointer. Exp Brain Res 190:11-30 
Rabini G, Altobelli E, Pavani F (2019) Interactions between egocentric and allocentric spatial coding of sounds revealed by a multisensory learning paradigm. Sci Rep 9(1):1-12

Rabini G, Lucin G, Pavani F (2020) Certain, but incorrect: on the relation between subjective certainty and accuracy in sound localisation. Exp Brain Res 238:727-739

Risoud M, Hanson JN, Gauvrit F et al (2019) Azimuthal sound source localization of various sound stimuli under different conditions. Eur Ann Otorhinolaryngol Head Neck Dis 137:21-29

Sabin AT, Macpherson EA, Middlebrooks JC (2005) Sound localization at near-threshold levels. Hear Res 39:1224-1231

Shub DE, Richards VM (2009) Psychophysical spectro-temporal receptive fields in an auditory task. Hear Res 251:1-9

Shub DE, Carr SP, Kong Y et al (2008) Discrimination and identification of azimuth using spectral shape. J Acoust Soc Am 124:3132-3141

Slattery WH 3rd, Middlebrooks JC (1994) Monaural sound localization: acute versus chronic unilateral impairment. Hear Res 75:38-46

Snapp HA, Holt FD, Liu X et al (2017) Comparison of speech-innoise and localization benefits in unilateral hearing loss subjects using contralateral routing of signal hearing aids or bone-anchored implants. Otol Neurotol 38:11-18

Souffi S, Nodal FR, Bajo VM et al (2021) When and how does the auditory cortex influence subcortical auditory structures? New insights about the roles of descending cortical projections. Front Neurosci. 15:690223

Stevens SS, Newman EB (1936) The localization of actual sources of sound. Am J Psychol 48(2):297-306

Strelnikov K, Rosito M, Barone P (2011) Effect of audiovisual training on monaural spatial hearing in horizontal plane. PLoS ONE 6(3): 18344

Thompson NJ, Kane SLG, Corbin NE, Canfarotta MW, Buss E (2020) Spatial hearing as a function of presentation level in moderateto-severe unilateral conductive hearing loss. Otol Neurotol 41:167-172
Valzolgher C, Campus C, Rabini G et al (2020) Updating spatial hearing abilities through multisensory and motor cues. Cognition 204:104409

Van Deun L, van Wieringen A, Scherf F et al (2010) Earlier intervention leads to better sound localization in children with bilateral cochlear implants. Audiol Neurootol 15:7-17

Wasmann JA, Janssen AM, Agterberg MJH (2020) A mobile sound localization setup. MethodsX 7:101131

Wightman FL, Kistler DJ (1997) Monaural sound localization revisited. J Acoust Soc Am 101:1050-1063

Wilska A (1938) Studies on directional hearing. English translation, Aalto University School of Science and Technology, Department of Signal Processing and Acoustics, 2010. PhD thesis originally published in German as Untersuchungen über das Richtungshören, University of Helsinki. http://legacy.spa.aalto.fi/publications/ WilskaThesis/

Wright BA, Zhang Y (2006) A review of learning with normal and altered sound-localization cues in human adults. Int $\mathrm{J}$ Audiol 45(Suppl 1):S92-98

Yang J, Wang Z, Huang M et al (2018) BoneBridge implantation in patients with single-sided deafness resulting from vestibular schwannoma resection: objective and subjective benefit evaluations. Acta Otolaryngol 138(10):877-885

Zirn S, Angermeier J, Arndt S et al (2019) Reducing the device delay mismatch can improve sound localization in bimodal cochlear implant/hearing-aid users. Trends Hear 23:2331216519843876

Zonooz B, Van Opstal AJ (2019) Differential adaptation in azimuth and elevation to acute monaural spatial hearing after training with visual feedback. eNeuro 1:6

Publisher's Note Springer Nature remains neutral with regard to jurisdictional claims in published maps and institutional affiliations.

\section{Authors and Affiliations}

\section{Tine Arras ${ }^{1} \cdot$ Hillary Snapp ${ }^{2} \cdot$ Anouk Sangen $^{1} \cdot$ Chantal Snels $^{3,4} \cdot$ Iris Kuntz $^{5} \cdot$ Tinne Theunen $^{6} \cdot$ Kiana Kheirkhah $^{7}$. Andrzej Zarowski ${ }^{6} \cdot$ Thomas Wesarg $^{5}$. Astrid van Wieringen ${ }^{1} \cdot$ Martijn J. H. Agterberg ${ }^{3,7}$}

Tine Arras

tine.arras@kuleuven.be

Hillary Snapp

hsnapp@med.miami.edu

Anouk Sangen

anouksangen@hotmail.com

Chantal Snels

chantalsnels@gmail.com

Iris Kuntz

iris.kuntz@uniklinik-freiburg.de

Tinne Theunen

tinne.theunen@gza.be

Kiana Kheirkhah

kianakheirkhahh@gmail.com

Andrzej Zarowski

andrzej.zarowski@me.com

Thomas Wesarg

thomas.wesarg@uniklinik-freiburg.de
Astrid van Wieringen

astrid.vanwieringen@kuleuven.be

1 Department of Neurosciences, Experimental ORL, KU

Leuven, University of Leuven, Leuven, Belgium

2 Department of Otolaryngology, University of Miami, Miami, FL, USA

3 Department of Otorhinolaryngology, Donders Institute for Brain, Cognition and Behaviour, Radboudumc, Nijmegen, The Netherlands

4 Department of Otorhinolaryngology, University Ghent, Ghent, Belgium

5 Department of Otorhinolaryngology-Head and Neck Surgery, Medical Center-University of Freiburg, Faculty of Medicine, University of Freiburg, Freiburg im Breisgau, Germany

6 ENT Department Sint-Augustinus Antwerp, European Institute For ORL, Antwerp, Belgium 
7 Department of Biophysics, Donders Institute for Brain, Cognition and Behaviour, Radboud University,
Heyendaalseweg 135, 6525 AJ Nijmegen, The Netherlands 